Research Paper

\title{
Self-assembled Photonic Crystals with Tunable Optical Properties
}

\author{
RAJESH V NAIR* \\ Laboratory for Nano-scale Optics and Meta-materials (LaNOM), Department of Physics, Indian Institute \\ of Technology (IIT), Ropar, Rupnagar, Punjab 140 001, India
}

(Received on 15 December 2015; Revised on 16 February 2016; Accepted on 22 March 2016)

\begin{abstract}
We have studied the tunable photonic stop gaps in three-dimensionally ordered photonic crystals in the visible and nearinfrared wavelength ranges. Structural analysis shows the (111) plane of the crystal with fewer imperfections. Angleresolved photonic stop gaps indicate the coupling between the Bragg waves diffracted by different crystal planes. The measured Bragg wave coupling is in good agreement with theoretical calculations. The new diffraction peak arises at the Bragg wave coupling is identified as from the $(-111)$ plane of the $f c c$ Brillouin zone. The modification of spontaneous emission of embedded quantum emitters inside the photonic crystals is studied using laser-induced emission measurements. We have achieved a suppression of $51 \%$ in the emission intensity at the stop gap wavelength. At higher excitation energy, strong band-edge induced mirrorless lasing is demonstrated. Our results have applications in wide variety of wave propagation in materials, solid state lighting, and nano-lasers.
\end{abstract}

Keywords: Photonic Crystals; Meta-materials; Spontaneous Emission; Nano-lasers; Quantum Electrodynamics

\section{Introduction}

There is a considerable interest in studying the lightmatter interactions in nanophotonic structures (Notomi, 2010; von Freymann, 2010). Photonic crystals or photonic band gap materials are a class of nanophotonic structure wherein the refractive index is periodically altered with a period of the order of wavelength of light (Yablonovitch, 1987; John, 1987). Because of the periodic variation in the refractive index, reflected light from each crystal planes can interfere constructively or destructively according to the Bragg condition for diffraction. This strongly depends on the wavelength of incident light, periodicity, and the refractive index contrast. Thus, when the Bragg condition is satisfied, a peak in reflectivity spectra is accompanied with a trough in transmission spectra and hence light is not able to propagate through the structure. This is the signature of photonic stop gap in the propagation direction (Lopez, 2003). When the photonic stop gaps in all the spatial direction occur at the same frequency for both polarization states of the incident light, a photonic band gap is formed
(Huisman et al., 2011). At the band gap frequencies light cannot couple to the structure and hence the photon density of states (DOS) is zero at the band gap frequencies. Therefore, photonic crystal structure is an ideal platform to tailor the light propagation and emission through engineering DOS (Yablonovitch, 1987). It is an extremely challenging task to measure, experimentally, the photonic band gap. Recently, a signature of the 3D photonic band gap has been achieved in diamond-like silicon inverse woodpile photonic crystals (Huisman et al., 2011). Even though the photonic band gap is extremely difficult to observe, photonic stop gap which exhibit only in particular directions of the light propagation is also useful in studying the light-matter interactions. This helps in exploring various physical aspects of light propagation and emission in photonic crystals using much simpler structures (Lopez, 2003).

Incident light with wavelengths within the photonic stop gap are forbidden to propagate inside the crystal. This is due to the unavailability of photonic states within the crystal at the stop gap wavelengths.

*Author for Correspondence: E-mail: rvnair@iitrprac.in 
Similarly, if light sources emit light within the crystal structure with emission wavelength equal to the stop gap wavelength, the photons cannot exit from the crystal due to stop gap. As a consequence of this, the allowed DOS at the stop gap wavelengths vanishes and it is enhanced near the band edges. Therefore, tailoring the DOS via engineering the photonic crystals can control the emission properties at any wavelength range for different polarization states of light (Vos et al., 2009; Lodahl et al., 2004). According to FermiGolden rule, absence of photonic DOS gives zero radiative decay rates which indicates an infinite lifetime for the excited atom or molecule. But the excited atom or molecule can decay through non-radiative decay channels (Lodahl et al., 2004). Such control on DOS has eminent opportunity in quantum electrodynamics and quantum information processing.

An important consequence of vanishing DOS is the inhibition of spontaneous emission (Yablonovitch, 1987; Vos et al., 2009). This inhibition of spontaneous emission at selected wavelengths can be exploited for achieving low-threshold lasing (Yablonovitch, 1987). For lasing to occur, according to the relation between Einstein spontaneous $(A)$ and stimulated $(B)$ emission coefficients, the spontaneous emission must be minimal so that the stimulated emission is enhanced for a given frequency. In any case, whether it is spontaneous or stimulated, if the emission is at the stop gap frequency, light cannot exit from photonic crystals and hence stop gap lasing is not possible. The enhancement of DOS in the wavelength range of stop gap can be achieved by creating intentional defects that effectively act as cavities with high quality factors in the otherwise periodic structure (Painter et al., 1999). This enhancement in DOS leads to an increased spontaneous emission rate when the cavity mode frequency is resonant with the emission frequency of emitters (John and Quang, 1994). This is one regime in photonic crystals to achieve low-threshold lasing by guiding the spontaneously emitted photons into the stimulated emission process. This is dictated using the $\beta$-parameter which is defined as the number of spontaneously emitted photons contributing to the stimulated emission process out of the total number of spontaneously emitted photons. A sought-after goal in nanophotonics research is to reach $\beta=1$ or the socalled thresholdless laser (Florescu et al., 2002). In a photonic crystal without cavities, an enhancement of DOS can also occur at the photonic band edge frequencies (John and Quang, 1994; Kopp et al., 1998; Nair et al., 2012; Nair et al., 2007). Near the band edge, the photonic bands become nearly flat resulting in a reduced group velocity for light so that the lightmatter interactions can be enhanced. The DOS is inversely proportional to the group velocity and therefore, it is enhanced at the edges.

In this paper, we report tunable photonic stop gaps in 3D ordered photonic crystals at the nearinfrared and visible wavelength ranges. The Bragg wave coupling occur at high angles of incidence of light is demonstrated. Our experimental results show well-resolved multiple Bragg peaks with high reflectivity values. We also compare our experimental results with theoretical calculations and the new stop gap observed in the coupling regime is assigned to the respective crystal plane. Further, laser-induced emission measurements show the suppression of emission intensity and at high pump energy, band-edge induced lasing is observed. We have used all-solid self-assembled photonic crystals made of Rhodamine dye doped colloids to investigate the modification of spontaneous emission of dyes in photonic crystals. Since the gain medium is doped into each building block, here the PS colloids, thus avoiding post infiltration of gain medium. \{In our samples, the gain medium is attached to each building blocks (PS colloids) and therefore the post-infiltration of gain medium is not required\}. This results in a uniform distribution of gain medium in every unit cells in the photonic crystal with zero gain in the surroundings.

\section{Experimental Details}

We have used commercially available polystyrene (PS) colloidal suspensions of sub-micron diameters for the synthesis of photonic crystals. We have also used Rhodamine B dyed PS micro-spheres for emission measurements. These micro-particles are charge stabilized to avoid the collision between the particles in the suspension. The well-known convective self-assembling method is used for the fabrication of photonic crystals. The experimental procedure is briefly explained here and details can be found elsewhere (Vos et al., 2009). We use PS spheres of diameter $D=803 \pm 26 \mathrm{~nm}, 266 \pm 6 \mathrm{~nm}$ as specified by the manufacturer (Microparticles $\mathrm{GmbH})$. The diameter of the dye-doped PS spheres is $295 \pm 8 \mathrm{~nm}$. We have used $1 \mathrm{wt} \%$ of the colloidal 
suspension in a cuvette with a clean glass substrate is placed in it and the whole assembly is kept in a temperature controlled oven for the growth of crystals. The temperature is tuned according to the sphere diameter so as to compete the Brownian motion of the particles in the suspension. Photonic crystals are grown in a time span of 5 days. The crystals are post-annealed at a temperature of $75^{\circ} \mathrm{C}$ for two hours to enhance its mechanical stability. The structural morphology is studied using a field-emission scanning electron microscope (FE-SEM). The samples are silver coated prior to FE-SEM characterization to avoid the charging effects. Optical reflectivity spectra are measured using a Perkin-Elmer lambda 950 spectrophotometer with Halogen lamp as the light source. The light beam is non-polarized and beam dimension is $5 \mathrm{~mm} \times 5 \mathrm{~mm}$. Silicon and lead sulfide detectors are used for collecting the light in the visible and near-infrared wavelength ranges, respectively. Laser induced emission studies are performed using a frequency-doubled Nd: YAG laser as a pumping source at a wavelength of $532 \mathrm{~nm}$ and pulse duration of $6 \mathrm{~ns}$. The light is focused on the sample using a lens of focal length $300 \mathrm{~mm}$ to an illumination spot of $\sim 1 \mathrm{~mm}$. The emitted light is focused onto the input slit of a monochromator and detected with a charge coupled detector.

\section{Structural and Optical Characterization}

The FE-SEM image of a photonic crystal made using PS spheres of diameter $803 \mathrm{~nm}$ is shown in Fig. 1(A). The image indicates very good ordering of spheres on the top surface and in the depth of the crystal. The hexagonal symmetry on the top surface represents the (111) plane of the face centered cubic $(f c c)$ lattice (Lopez, 2003). The signature of photonic stop gap is probed through the reflectivity and transmittance measurements. Photonic stop gap measured at nearnormal incidence of light $\left(\theta \sim 8^{\circ}\right)$ from the (111) plane is shown in Fig. 1(B). A peak in reflectivity spectra (black line) centered at $1750 \mathrm{~nm}\left(a \lambda_{c}=0.65, a\right.$ is the $f c c$ lattice constant and $\lambda_{c}$ is the stop gap wavelength) with a peak reflectance of $57 \%$ is accompanied by a trough in transmission spectra (red line) at the same wavelength with a transmittance of $18 \%$. This is the stop gap signature. The experimentally observed stop gap frequency is in good agreement with the photonic band structure calculations (Galisteo-Lopez et al., 2003).
The photonic strength $(S)$ or the full width at half maximum of the stop gap is estimated to be $6.57 \%$. The calculated photonic strength from photonic band structure is $6.50 \%$ (Bechger et al., 2005). The close agreement between the estimated and the calculated values indicate the superior optical quality of our photonic crystals. The Bragg length $\left(L_{B}\right)$, i.e., the distance through which light propagate at the stop gap wavelength before it is completely attenuated, can be calculated using the formula (Bechger et al., 2005), $L_{B}=2 d_{h k l} / \pi S$, where $d_{h k l}$ is the distance between the crystal planes in the $[h k l]$ direction. The measured $L_{B}$ along the [111] direction is $6 \mu \mathrm{m}$. The Fabry-Perot (F-P) oscillations (inset in Fig. 1(B)) are observed on either side of stop gap indicates_uniform thickness of the crystal and also indicates well ordering of each layer in the depth of photonic crystal. The measured thickness $(t)$ of the photonic crystal from the F-P fringes using the formula (Romanov et al., 2007), $t=1239 /\left(2 \times n_{\text {eff }} \times \Delta E\right)$, where $n_{\text {eff }}$ is the effective refractive index and $\ddot{A} E$ is the difference in energy positions in $e V$ of two consecutive troughs in the F-P oscillation. In the long-wavelength side of the stop gap, we estimate $t=14 \mu \mathrm{m}\left(\sim 2.4 L_{B}\right)$ which corresponds to 22 ordered layers. $t \sim 14 \mu \mathrm{m}(\sim 22$ ordered layers) and $t \sim 2.4 L_{B}$. This shows that the finite size effects are eliminated in our photonic crystals. This large number of ordered layers together with less defects and disorder in our photonic crystals enable us to probe stop gaps at high angles of incidence (Nair and Vijaya, 2007).

\section{Tunable Photonic Stop Gaps}

Angle-resolved reflectivity spectra provide information about photonic stop gaps at different angles of incidence of light. The photonic stop gap wavelength at different angles of incidence can be estimated using the modified Bragg's law for photonic crystals (Baryshev et al., 2007)

$$
\lambda_{h k l}=2 \times n_{e f f} \times d_{h k l} \times \cos \left(\alpha-\sin ^{-1}\left(\frac{1}{n_{e f f}} \sin \theta\right)\right)
$$

where $\lambda_{h k l}$ is the Bragg wavelength diffracted from $(h k l)$ crystal plane, $d_{h k l}$ is the distance between the $(h k l)$ crystal planes, $n_{\text {eff }}$ is the effective refractive index of the photonic crystal, $\lambda$ is the angle between $(h k l)$ and the (111) plane, and $\theta$ is the external angle of incidence. The calculations are done using an 
A
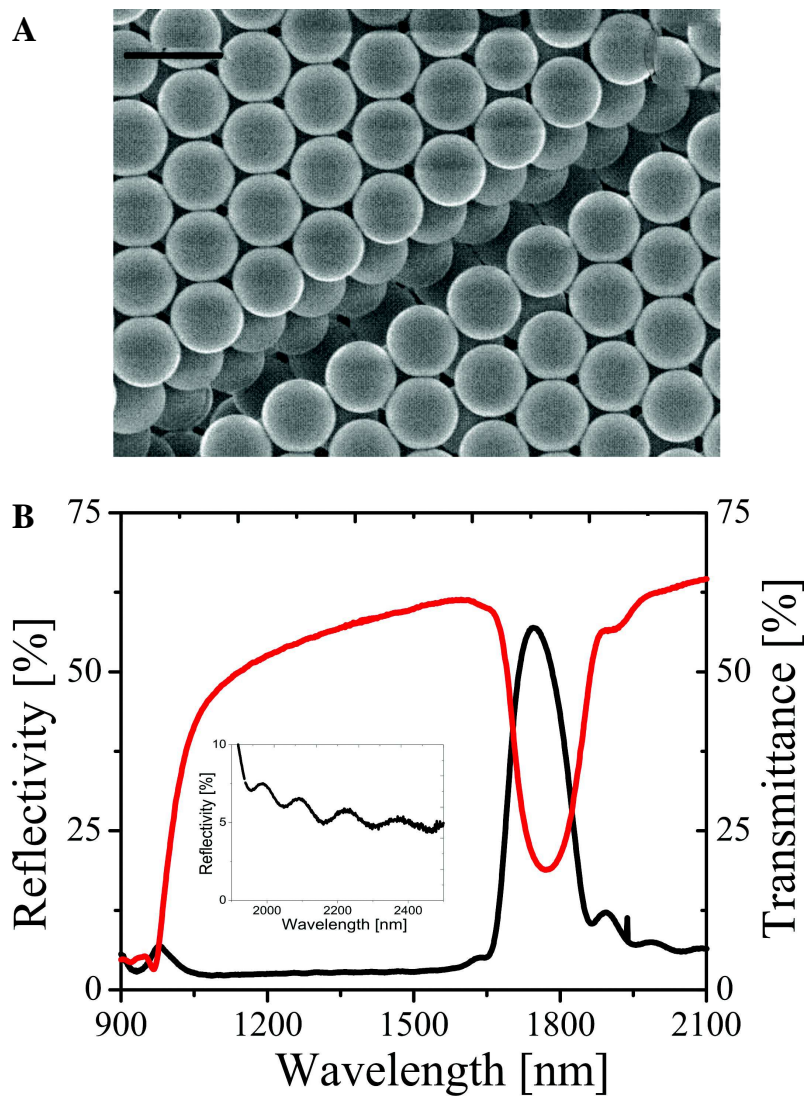

Fig. 1: (A) FE-SEM image of the photonic crystal made with PS spheres of diameter $803 \mathrm{~nm}$. The image confirms good ordering on the surface and in the depth of the sample. The hexagonal symmetry on the surface represents the (111) plane of the $f c c$ lattice. Scale bar is $1 \mu \mathrm{m}$. (B) Reflectivity (black line) and transmittance (red line) for a photonic crystal composed of PS spheres of diameter $803 \mathrm{~nm}$ shows the stop gap at $1750 \mathrm{~nm}$. Peak in reflectivity spectra aggress with the trough in transmission spectra which is the signature of photonic stop gap. Inset shows the FabryPerot oscillations on the long wavelength side of the stop gap

effective sphere diameter $\left(D_{\text {eff }}\right)$ of $750 \mathrm{~nm}$ which is obtained by fitting the measured stop gap wavelengths from the (111) plane with Eq. (1) for $\theta \leq 45^{\circ}$. It should be noted that the $D_{\text {eff }}$ is slightly less than that specified by the manufacturer $(803 \pm 26 \mathrm{~nm})$. In all the analysis, we use the sphere diameter of $750 \mathrm{~nm}, d_{111}=0.816 D_{\text {eff }}$ and $n_{\text {eff }}=1.44$ for PS photonic crystal.

The measured angle-resolved photonic stop gap from the (111) plane of the crystal is shown in Fig. 2. With increase in $\theta$ value, the photonic stop gap shifts towards the shorter wavelength region. The peak
A

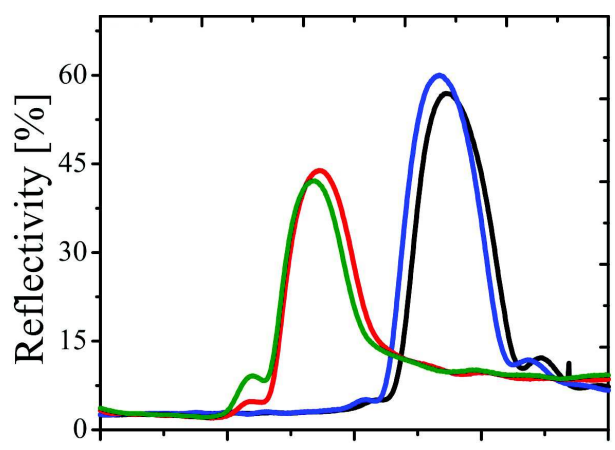

B

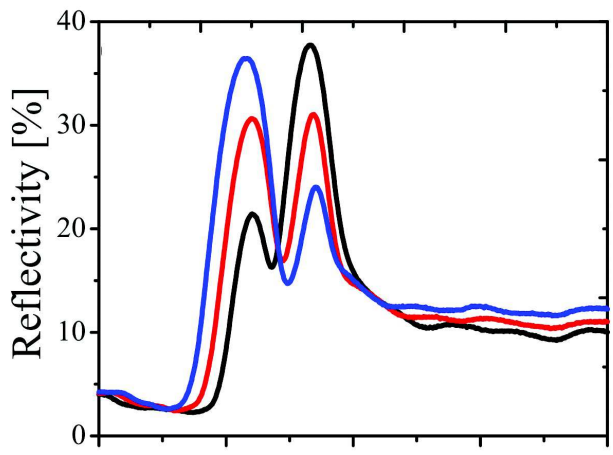

C

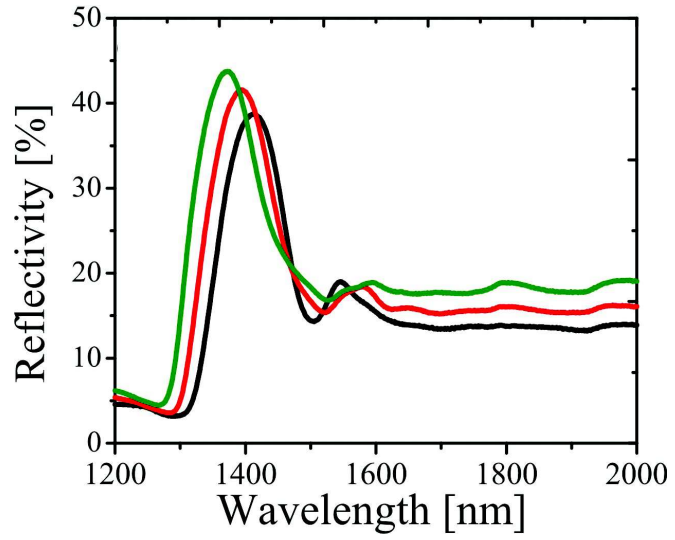

Fig. 2: (A) Photonic stop gap at angle of incidence of $8^{\circ}$ [black line], $15^{\circ}$ [blue line], $45^{\circ}$ [red line], and $47^{\circ}$ [green line]. With increase in angle of incidence, stop gap shifts towards the shorter wavelengths. At angles of incidence of $45^{\circ}$ and $47^{\circ}$, a new reflectivity peak starts appearing near the low-wavelength edge of the stop gap. (B) Photonic stop gap at angles of incidence of $50^{\circ}$ [black line], $5^{\circ}$ [red line], and at $56^{\circ}$ [blue line]. At $53^{\circ}$ incidence, the two diffraction peaks are well separated with equal reflectivity values. (C) Photonic stop gap at angles of incidence of $59^{\circ}$ [black line], $62^{\circ}$ [red line], and at $65^{\circ}$ [green line]. Both diffraction peaks are shifted in the opposite direction

reflectivity and the width of the (111) reflectivity peak remains unchanged till $\theta \leq 45^{\circ}$. For $\theta>45^{\circ}$, peak reflectivity is started decreasing and a new peak originate at the short wavelength band edge. Starting at $\theta=47^{\circ}$, the new peak become more intensified and 
shifts towards the higher wavelength region of (111) stop gap. The peak reflectivity of (111) stop gap is constantly decreasing and that the reflectivity of new peak keeps on increasing. For $\theta=50^{\circ}$, the peak reflectivity of (111) stop gap reduces marginally and that the reflectivity of new peak increases. At $\theta=$ $53^{\circ}$ (red line in Fig. 2(B)), the two reflectivity peaks are equally separated. They have the same peak reflectance and same width. The two Bragg waves are excited simultaneously inside the photonic crystal at this $\theta$ value. Their scattering strengths are equal and therefore they diffract with equal intensity. This is the avoided crossing regime wherein two Bragg waves are excited simultaneously inside the crystal and they repel each other. The two diffracted waves peaks are separated by $107 \mathrm{~nm}$ and are coupled (van Drieland Vos, 2000). At $\theta=56^{\circ}$, strength of (111) peak increases whereas the strength of the new peak is started decreasing and both peaks shift in the opposite directions. With further increase in $\theta$, new reflectivity peak moves towards the higher wavelength side whereas the (111) reflectivity peak shifts towards the lower wavelength side. Also, the reflectivity value of (111) peak peak reflectivity of (111) peak increases whereas that for the new reflectivity peak decreases. This shows that the energy is continuously exchanged between the two reflectivity peaks from the moment the new reflectivity peak started appearing at $47^{\circ}$.

The equal exchange of energy occurs at the avoided crossing region. The phenomena can be qualitatively visualized as follows: When $\theta$ crosses $45^{\circ}$, light diffracted by the crystal planes in the depth of the photonic crystal is re-directed to other crystal planes which in-turn re-diffract the light along the specular direction together with (111) diffraction peak. At specific $\theta$ value, the diffraction strength of both the peaks is equal. The two diffracted beams are constantly exchanging the energy between them. The peak wavelength depends on the angle of incidence of light which signifies that the observed phenomena are from the depth of the crystal and not due to any surface effects of 3D photonic crystals.

\section{Bragg Wave Coupling}

With increase in $\theta$ value, one diffraction peak shifts towards the lower wavelength region whereas the other diffraction peak shifts towards the higher wavelength region. Fig. 3(A) shows the evolution of (111) stop gap (closed symbols) and the new $(h k l)$ plane (open symbols) for a PS photonic crystal with $D_{\text {eff }}=750 \mathrm{~nm}$. The stop gap wavelengths are extracted through multiple Gaussian fittings to the measured reflectivity spectra. In Fig. 3(A), we have calculated the Bragg wavelength for two important planes in the $f c c$ Brillouin zone, namely, (111) [black line], and $(-111)$ [blue line] planes using Eq. (1). This corresponds to the $\Gamma-L$ and $\Gamma-K$ directions in the $f c c$ Brillouin zone. It is interesting to note the crossing of (111) and (-111) stop gap wavelengths around $\theta=$ $55^{\circ}$. Because of the crossing of these bands, for $\theta=$ $55^{\circ}$, the (111) stop gap is split into two peaks and they shift in opposite directions with further increase in $\theta$. This stop gap shift in the opposite direction is also shown in our theoretical calculations. Our experimental results are in good agreement with band crossing calculated using Eq. (1). When the external angle of incidence increases, the internal angle of incidence for the plane responsible for the diffraction peak increases for the (111) stop gap while it decreases for the new (-111) diffraction peak and both peaks shift in opposite directions. It is very important to note that, in our photonic crystals the finite size effects are eliminated as $t \sim 2.4 L_{B}$ and therefore, Bragg wave coupling shows extremely well-resolved diffraction peaks. We also compare our experimentally observed reflectivity peak wavelengths to the calculated photonic band structure reported in literature on similar photonic crystal structures (Pavarini et al., 2005). The new reflectivity peak appears near the crossing point at a reduced frequency of $a / \lambda_{c}=0.70$ in the measurements and that obtained from the calculated band structure is at $a / \lambda_{c}=0.73$ for wave vector near the $K$-point. This indicates that the Bragg wave coupling occur at the $K$-point. Therefore, the new reflectivity peak which appears near the crossing point is from the (-111) plane in the $f c c$ Brillouin zone and the incident wave vector moves towards the $K$-point and the wave vector trajectory is $\Gamma-L-K-L_{l}$ in the $f c c$ Brillouin zone (Baryshev et al., 2007). Also a closer look into Fig. 3(A) indicates that when the Bragg wave coupling begins, the (111) stop gap is repelled from the calculated stop gap wavelength curve which indicates an avoided crossing of bands. The very good optical quality of the photonic crystals shows multiple Bragg diffraction over tens of degree. Also, in the avoided crossing region, stop gap 
wavelengths are nearly the same due to the flattening of photonic bands (van Drieland and Vos, 2000).

In order to demonstrate the Bragg wave coupling in the visible wavelength region, we did experiments with self-assembled PS photonic crystal with $D_{e f f}$ of $260 \mathrm{~nm}$ [obtained from the Bragg's law fit to the stop gap wavelengths for $\theta \geq 45^{\circ}$. This gives us stop gap in the visible region with a stop gap wavelength $\left(\lambda_{c}\right)$ of $605 \mathrm{~nm}$. The measured reflectivity spectra are similar to those shown for the near-infrared wavelength region [see Fig. 2]. The measured and calculated stop gap wavelengths for the (111) and (111) planes are given in Fig. 3(b). Closed symbols
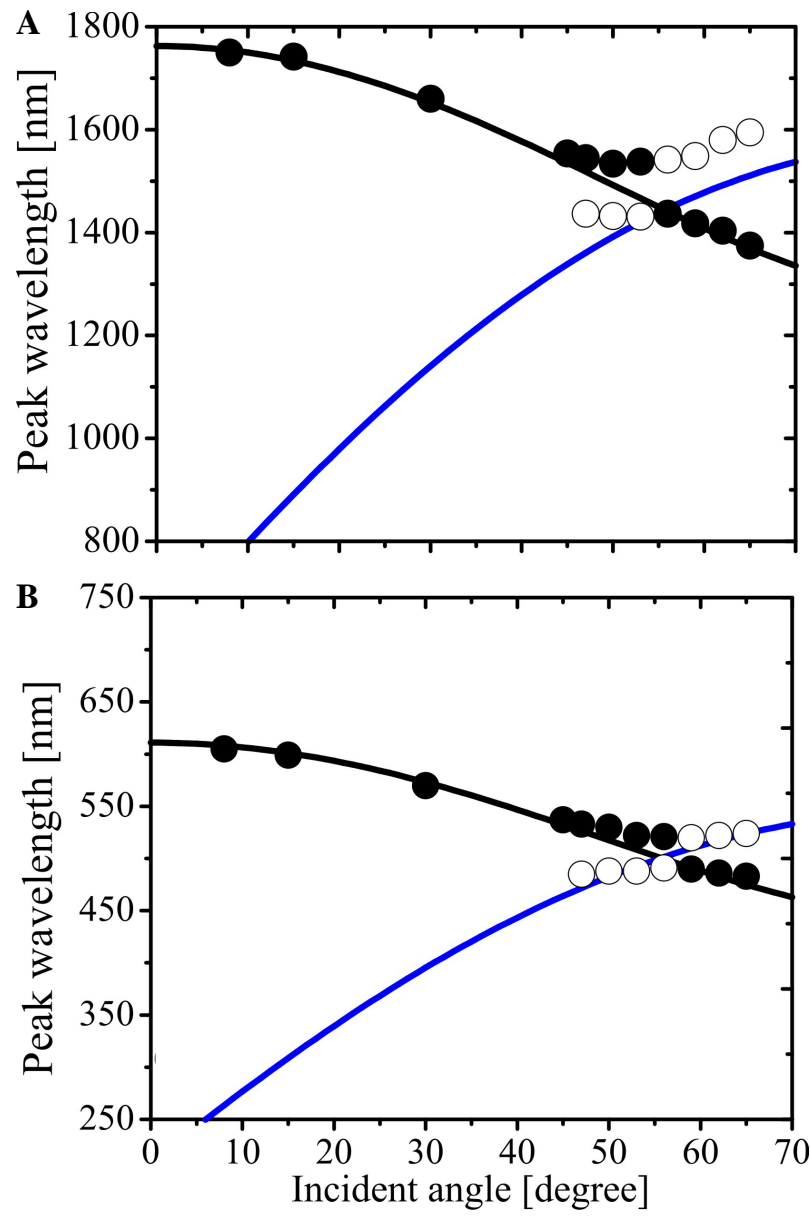

Fig. 3: Calculated stop gap wavelengths for two different crystal planes in the $f c c$ Brillouin zone, such as, (111) [black line], and (-111) [blue line] along with experimental stop gap wavelengths as a function of $\theta$ for PS photonic crystal with $D_{\text {eff }}=750 \mathrm{~nm}$ (A) and $D_{\text {eff }}$ $=260 \mathrm{~nm}(\mathrm{~B})$, respectively. Closed symbols shows the (111) stop gap and open symbols represents the (-111) stop gap wavelengths. Bragg wave coupling occurs at $\boldsymbol{\theta}=\mathbf{5 5}^{\circ}$ show the (111) stop gap and open symbols represent the (-111) stop gap at different $\theta$. The Bragg wave coupling occurs at $\theta=55^{\circ}$ with a well-resolved Bragg diffraction peak having equal reflectivity. The wavelength region where the Bragg wave coupling occurs is around $500 \mathrm{~nm}$ in Fig. 3(B) as compared to that at $1500 \mathrm{~nm}$ in Fig. 3(A). This is due to the difference in lattice constants even though the index contrast is same for the two samples. Thus the wavelength region of avoided crossing is proportional to the lattice constant. This is another confirmation that Bragg diffraction is the basis of observed phenomena (Nair and Jagatap, 2012).

\section{Spontaneous Emission Control}

Fig. 4(A) depicts the emission spectra recorded around $23^{\circ}$ to the [111] direction from the dyed-PS photonic crystals using a low excitation energy ( 0.35 $\mathrm{mJ})$. Solid line represents the emission spectra for photonic crystals with sphere diameter $295 \mathrm{~nm}$ (dyedPS295) and dashed line represents the emission from photonic crystals with sphere diameter $617 \mathrm{~nm}$ (dyedPS617). Here the dyed-PS617 is the reference sample and each emission curve is normalized to its peak value. The pump energy is kept low in order to avoid the participation of any gain effects in the emission measurements. It is known that the emission features of dyes are sensitive to the solvents, and hence the emission from a liquid solution can differ from the dye-doped polymer. Therefore, a reference sample using the same dye doped into the same polymer spheres needs to be utilized to facilitate a proper comparison. The photonic stop gap of dyedPS617 is centered at $1350 \mathrm{~nm}$, and hence the dye emission is not affected by the photonic stop gap. Therefore, we use dyed-PS617 as a proper reference crystal as it possess the same crystal symmetry, filling fraction, and the same dye is doped within the spheres. Therefore, the measured emission from the dyedPS617 sample is taken as the intrinsic dye emission. The emission spectra measured from dyed-PS295 shows inhibition in emission intensity in the wavelength range of 585 to $610 \mathrm{~nm}$ and also the emission maximum is blue shifted.

The emission spectra (dotted line) measured from dyed-PS617 is centered at $600 \mathrm{~nm}$ as shown in Fig. 4(A). The emission intensity abruptly reduces to zero on the red side of emission spectrum due to the 
limited spectral range of our measurement set up. The emission spectrum (solid line) from dyed-PS295 in Fig. 4(A) shows two interesting features. One is the suppression of emission intensity in a wavelength range of 590 to $605 \mathrm{~nm}$ and second, the emission maximum is blue-shifted. Fig. 4(B) shows the measured intensity ratio (solid line) between the dyedPS295 and dyed-PS617 at $\theta=23^{\circ}$; wherein the suppression in emission intensity is more evident. The photonic stop gap (squares) along the [111] direction at the same $\theta$ value is also shown in Fig. 4(B). The intensity ratio is expected to be unity for samples in the absence of any stop gap effect. But in Fig. 4(B), the intensity ratio shows a clear suppression in the wavelength range of 595 to $610 \mathrm{~nm}$, which overlaps with the photonic stop gap along [111] direction. This is due to the re-distribution of DOS (Nikolaev et al., 2005). The attenuation in the emission intensity at the stop gap is quantified by $\Delta I / I_{0}$ and is estimated to be $51 \%$. The peak reflectance value is also $\sim 50 \%$ within the stop gap using external light sources. The stop gap effect for externally incident light waves (square symbols) and that for internally excited light sources (solid line) are clearly observed in Fig. 4(B). Such a strong modification of emission characteristics in allsolid self-assembled photonic crystal is due to the superior quality of the ordering present in our samples. We observe in Fig. 4(B) that the intensity ratio is above unity in the blue side of the photonic stop gap. This shows that more photons are emitted in the blue side of the stop gap when the emission is suppressed within the stop gap. The excess emission can be understood as follows. When a source is excited inside the photonic crystal, the emitted light is diffuse and propagates in all possible directions except in the stop gap direction. This diffuse propagating light leaves the crystal at the blue side of the photonic stop gap. Such an enhancement is understood in terms of an escape function (Koenderink and Vos, 2005), which essentially quantifies the escape probability of photons in all directions. This escape function which accounts for the diffuse nature of emitted light shows large enhancement near the blue side of the stop gap, when the emission is suppressed within the stop gap, as compared to the red side.

\section{Photonic Band-edge Lasing}

The emission spectra measured from dyed-PS617 and dyed-PS295 samples at different excitation energy
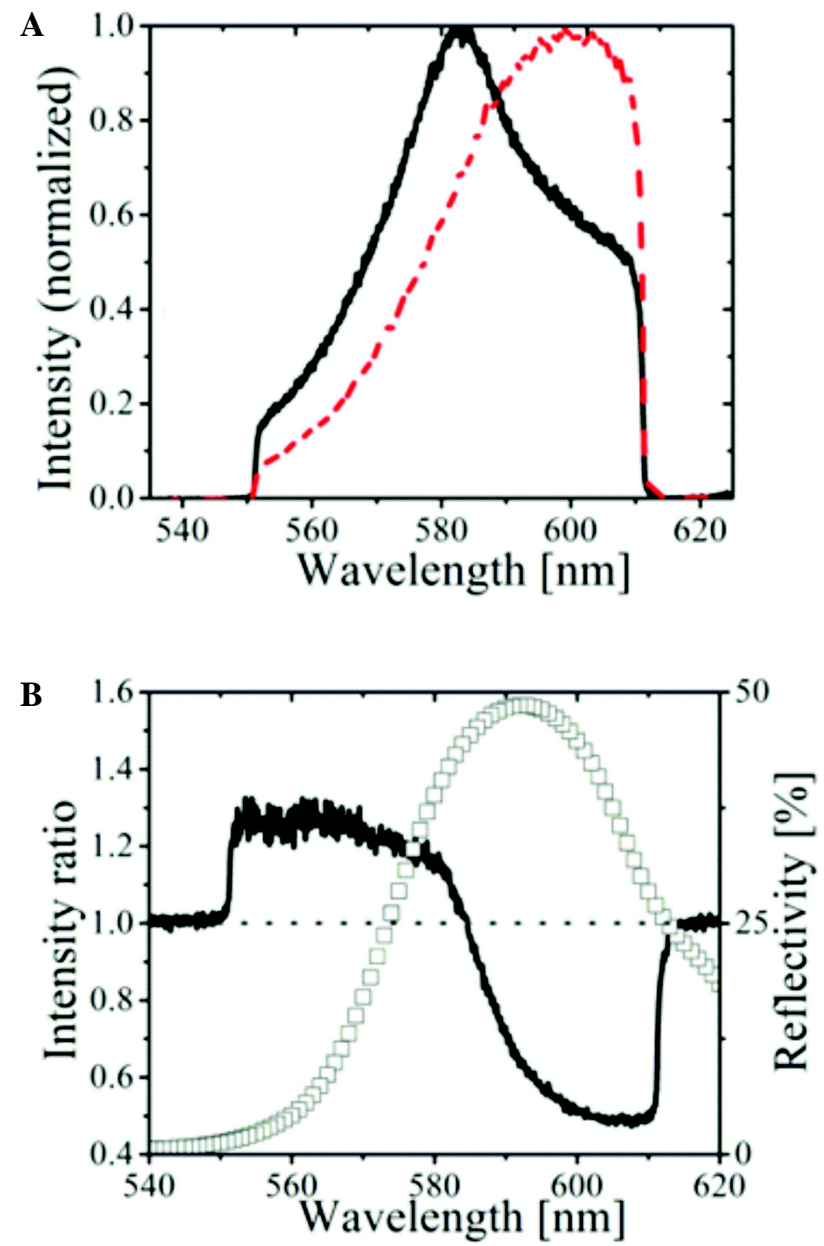

Fig. 4: (A) Emission spectra measured from the photonic crystals composed of dyed-PS spheres of diameter $295 \mathrm{~nm}$ (solid line) and $617 \mathrm{~nm}$ (dashed line). Emission spectra for photonic crystals of sphere diameter $295 \mathrm{~nm}$ show an abrupt decrease in emission intensity around $595 \mathrm{~nm}$ due to photonic stop gap. The emission spectra for photonic crystals of sphere diameter $617 \mathrm{~nm}$ do not show any changes in intensity. (B) The intensity ratio (solid line) between the emission spectra of photonic crystals with sphere diameter of $295 \mathrm{~nm}$ and $617 \mathrm{~nm}$ together with corresponding stop gap (open squares) shows the suppression of spontaneous emission in the stop gap wavelength ranges. The trough in intensity ratio is in good agreement with peak in reflectivity spectra. The intensity ratio is above unity in the blue side of the photonic stop gap

are given in Fig. 5(A) and 5(B), respectively. With increase in excitation energy the emission intensity increases for dyed-PS617sample as seen in Fig. 5(A). However, the dyed-PS295 sample shows the origin of a strong narrow peak at the low-frequency (high 
wavelength) band edge. This peak is absent for the dyed-PS617 sample at any excitation energy as seen in Fig. 5(A). The measured peak for dyed-PS295 at $606.41 \pm 0.19 \mathrm{~nm}$ with a narrow width of $0.78 \pm 0.13$ $\mathrm{nm}$ represents band edge lasing peak, and occurs at
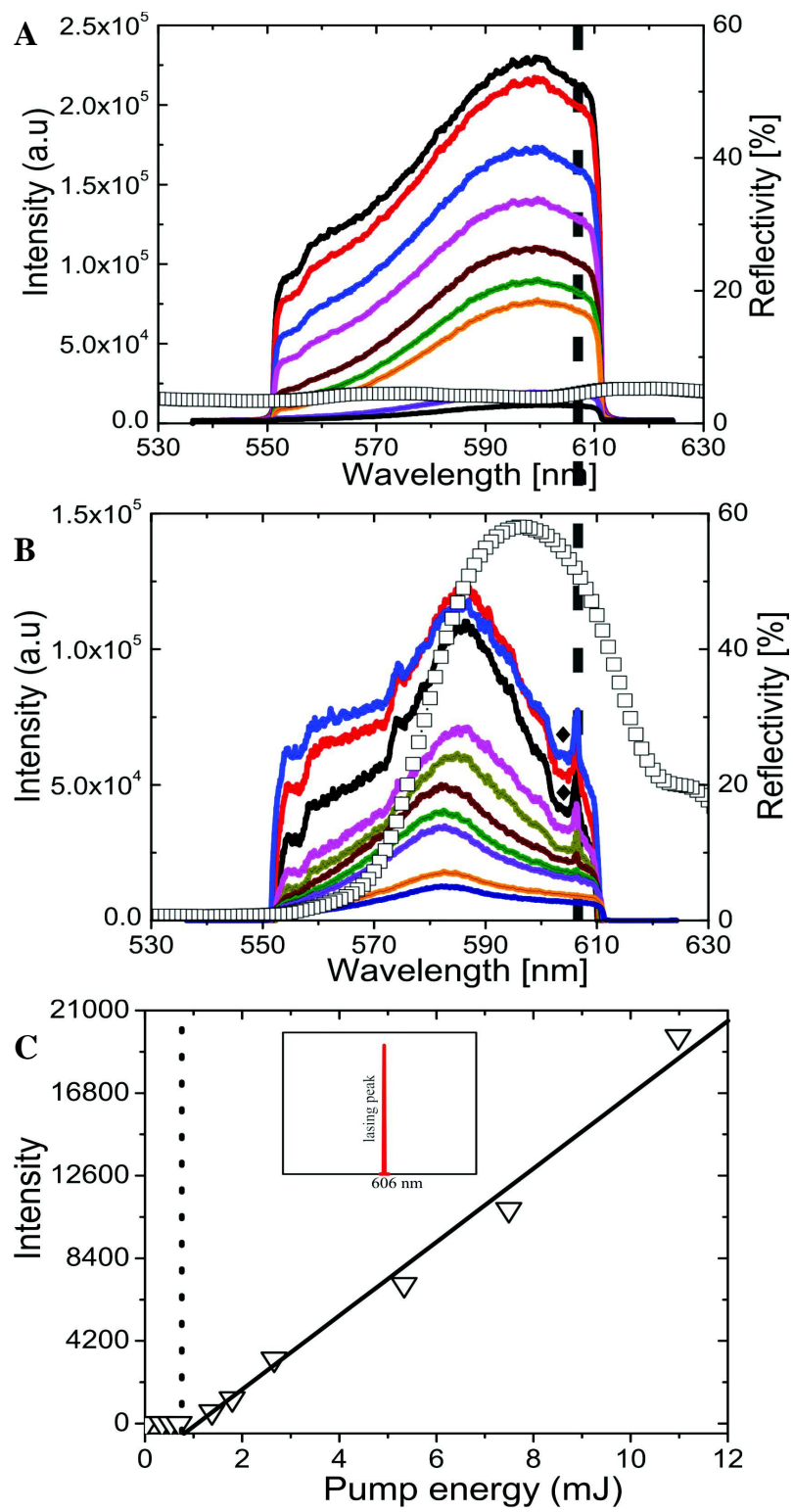

Fig. 5: Laser induced emission spectra at different excitation energy for (A) dyed-PS617 sample and (B) dyed-PS295 sample. In (A) with increase in excitation energy emission intensity increases whereas in (B), at higher excitation energy, a narrow peak originates near the long wavelength band edge. In both (A) and (B) symbols represents the stop gap in the investigated spectral range. (C) Laser threshold curve at $606 \mathrm{~nm}$ shows lasing occurs at a threshold of $0.7 \mathrm{~mJ}$. Inset shows the lasing spectra above threshold the long wavelength photonic band edge. It is also seen that irrespective of the value of excitation energy the spontaneous emission is suppressed within the stop gap and hence no stop gap lasing is possible. When the lasing peak starts builds up near the long wavelength band edge, the spontaneous emission at the stop gap wavelength shows a clear suppression within the stop gap (marked with diamond symbol).

The enhancement of DOS occurs at both lowand high-wavelength band edges. However, we did not observe any lasing peak near the low-wavelength band edge, irrespective of the incident pump energy, as seen in Fig. 5(b). The electric field distribution at the long wavelength band edge concentrates its energy in the high-index material (gain medium in the present case) and the short wavelength band edge concentrates the energy in the low-index material (air in the present case). Hence only low-frequency (highwavelength) band edge experiences the gain and consequently the lasing occurs only at the lowfrequency band edge. The threshold curve is plotted in Fig. 5(C) for the lasing peak at $606 \mathrm{~nm}$. A clear threshold at $0.7 \mathrm{~mJ}$ is observed, wherein the stimulated emission overcomes the spontaneous emission. The inset shows the emission spectrum above threshold at $606 \mathrm{~nm}$.

\section{Conclusions}

To conclude, we have shown tunable photonic stop gaps in the visible and near-infrared wavelength ranges. Our experimentally observed photonic stop gap from the (111) plane of photonic crystals is in good agreement with band structure calculations. Angle-resolved photonic stop gap shows the coupling between the Bragg waves diffracted by different crystals planes other than the (111) plane. The observed Bragg wave coupling is in good agreement with theoretical calculations. The new diffraction peak arises at higher angle of incidence is from the (-111) plane. The emission properties of the Rhodamine B dye embedded in a photonic crystal is analyzed using a photonic crystal made of dyed polystyrene spheres. Laser induced emission experiments show the suppression in emission intensity of $\sim 51 \%$ in the photonic stop gap. At higher excitation energies; we have demonstrated the signature of band-edgeinduced lasing characteristics. The origin of lasing is explained as due to the enhanced density of photon 
states and field distribution at the band edge frequencies. Our all-solid photonic crystal band edge laser is a promising candidate for nanophotonics applications.

\section{References}

Baryshev A V, Khanikaev A B, Fujikawa R, Uchida H and Inoue M (2007) Polarized light coupling to thin air-silica opal films grown by vertical deposition Phys Rev B 76014305 $1-9$

Bechger L, Lodahl P and Vos W L (2005) Directional Fluorescence Spectra of Laser Dye in opal and inverse opal Photonic Crystals J Phys Chem B 109 9980-9988

Florescu L, Busch K and John S (2002) Semiclassical theory of lasing in photonic crystals J Opt Soc Am B 19 2215-2223

Galisteo-Lopez J F, Palacios-Lidon E, Castillo-Martý'nez E and Lopez C (2003) Optical Study of pseudogap in thickness and orientation controlled artificial opals Phys Rev B 68 $115109-1-8$

Huisman S R, Nair R V, Woldering L A, Leistikow M D, Mosk A $P$ and Vos W L (2011) Signature of a three dimensional photonic band gap observed on silicon inverse woodpile photonic crystals Phys Rev B 83 205313-1-7

John S (1987) Strong localization of photons in certain disordered dielectric super lattices Phys Rev Lett 58 2486-2489

John S and Quang T (1994) Spontaneous emission near the edge of a photonic band gap Phys Rev A 50 1764-1769

Koenderink A F and Vos W L (2003) Light exiting from real photonic band gap crystals is diffuse and strongly directional Phys Rev Lett 91 213902-1-4

Kopp V I, Fan B, Vithana H K M and Genack A Z (1998) Lowthreshold lasing at the edge of a photonic stop band in cholesteric liquid crystal Opt Lett 23 1707-1709

Lodahl P, Floris van Driel A, Nikolaev I S, Irman A, Overgaag K, Vanmaekelbergh D and Vos W L (2004) Controlling the dynamics of spontaneous emission from quantum dots by photonic crystals Nature 430 654-657

Lopez C (2003) Material aspects of photonic crystals Adv Mater 15 1679-1704

Nair R V and Jagatap B N (2012) Bragg coupling in self-assembled

\section{Acknowledgements}

The author thanks IIT Ropar for all the support and Board of Research in Nuclear Science (BRNS), Govt. of India, for Dr. K S Krishnan Research Fellowship. Dr. B N Jagatap, Director, Chemistry Group, BARC is gratefully acknowledged for the support. Dr. S Mujumdar, TIFR Mumbai is also acknowledged for help with emission measurements.

opal photonic crystals Phys Rev A 85 013829-1-10

Nair R V and Vijaya R (2007) Infiltration of polymeric Photonic Crystal by the sol-gel process J Phys D: Appl Phys 40 990-997

Nair R V and Vijaya R (2007) Observation of higher order diffraction features in self assembled photonic crystals Phys Rev A 76 053805-1-7

Nair R V, Tiwari A K, Mujumdar S and Jagatap B N (2012) Photonic-band-edge-induced lasing in self-assembled dyeactivated photonic crystals Phys Rev A 85 023844-1-7

Nikolaev I S, Lodahl P and Vos W L (2005) Quantitative analysis of directional spontaneous emission spectra from light sources in photonic crystals Phys Rev B 71 053813-1-10

Notomi M (2010) Manipulating light with strongly modulated photonic crystals Rep Prog Phys 73 096501-1-57

Painter O, Lee R K, Scherer A, Yariv A, O'Brien J D, Dapkus P D and Kim I (1999) Two-dimensional photonic band-gap defect mode laser Science 284 1819-1821

Pavarini E, Andreani L C, Soci C, Galli M, Marabelli F and Comoretto D (2005) Band structure and optical properties of opal photonic crystals Phys Rev 72 045102-1-9

Romanov S G et al. (2007) Erasing Diffraction orders : Opal versus Langmuir Blodgettcolloidal crystals Appl Phys Lett 90 133101-1-3

vanDriel H M and Vos W L (2000) Multiple Bragg wave coupling in photonic band gap crystals Phys Rev B $629872-9875$

von Freymann G et al. (2010) Three-dimensional nanostructure for photonics Adv Funct Mater 20 1038-1052

Vos W L, Koenderink AF and Nikolaev I S (2009) Orientationdependent spontaneous emission rates of a two-level quantum emitter in any nanophotonic environment Phys Rev A 80 053802-1-7

Yablonovitch E (1987) Inhibition of spontaneous emission in solid state physics and electronics Phys Rev Lett 58 20592062. 